\title{
Phénomène d'érosion-corrosion dans les pompes alimentaires et préalimentaires des générateurs de vapeur
}

\author{
Erosion and corrosion effects \\ in main and preliminary feed pumps for steam generators
}

\author{
Léon Randrianarivo
}

Département Machines et Structures, Direction des Études et Recherches, Électricité de France (Chatou)

\section{Introduction}

Depuis quelques années, on découvre, principalement dans les corps des pompes alimentaires et préalimentaires des générateurs de vapeur des tranches thermiques classiques d'Electricité de France, des dégradations qui affectent certaines zones, soumises à des vitesses relatives métal-fluide élevées, non consécutives à un phénomène du type érosion par cavitation ou par abrasion et qui sont la manifestation de l'érosioncorrosion.

Ces dégradations peuvent avoir des conséquences graves dans la mesure où leur évolution dans le temps semble être très rapide. Des expertises récentes faites sur des corps de pompes préalimentaires et alimentaires de quelques centrales thermiques classiques d'Electricité de France ont, en effet, mis en évidence des pertes d'épaisseur pouvant atteindre localement $15 \mathrm{~mm}$ et plus après environ 30000 à 40000 heures de fonctionnement, et ceci, dans des conditions de température de l'ordre de 160 à $180^{\circ} \mathrm{C}$, de $p H$ de 8,5 à 9 (à $25^{\circ} \mathrm{C}$ ) et de vitesses d'écoulement de 12 à $23 \mathrm{~m} / \mathrm{s}$.

Devant l'importance de ces dégradations et l'évolution sans cesse croissante, dans les projets en étude, des caractéristiques des pompes alimentaires, la Direction des Etudes et Recherches, en collaboration avec les constructeurs de pompes, consacre actuellement une étude au phénomène d'érosion-corrosion dans les conditions générales de fonctionnement de ce type de matériel.

Cette étude repose simultanément sur :

- Une série d'observations des dégradations imputables à l'érosion-corrosion dans les pompes actuellement en service, suivie d'une analyse critique des phénomènes observés afin de pouvoir dégager les paramètres influents et de tenter une interprétation théorique de ces phénomènes.
- Une étude expérimentale du phénomène d'érosioncorrosion dans l'eau chaude désaérée à grande vitesse d'écoulement ( $80 \mathrm{~m} / \mathrm{s}$ et plus) par l'intermédiaire de deux boucles d'essai dont nous donnons ci-après une description sommaire.

Rôle et description succints des pompes alimentaires et préalimentaires

Les pompes alimentaires aspirent l'eau de la bâche alimentaire pour la refouler dans le réservoir de la chaudière en traversant les réchauffeurs HP du poste d'eau et l'économiseur du générateur de vapeur (voir fig. 1).

Les pompes alimentaires sont du type centrifuge, multicellulaire, à axe horizontal, d'un débit horaire d'environ 200 tonnes pour les tranches thermiques classiques de $125 \mathrm{MW}$, de 800 tonnes pour celles de $250 \mathrm{MW}$ et de 2000 tonnes pour celles de $600 \mathrm{MW}$. La pompe comprend (voir fig. 2) :

- Un corps cylindrique en acier forgé sur lequel sont soudées les tubulures d'aspiration et de refoulement. Celles-ci sont en général dans le plan vertical de l'axe. Le corps est obturé des deux côtés par des fonds ou flasques, en acier forgé également, tenus par des boulons en acier allié. Le fond d'aspiration forme guide pour l'entrée d'eau.

- Un empilage de diffuseurs et de pièces conductrices se trouve à l'intérieur du corps et porte le nom de bloc interne. Ces pièces sont en acier à $13 \%$ de chrome, elles sont maintenues ensemble par des tiges d'assemblage dans la plupart des cas.

Pour les pompes alimentaires plein débit des tranches de $250 \mathrm{MW}$ et de $600 \mathrm{MW}$, il devient impossible de les alimenter par l'eau venant directement de la bâche alimentaire, l'insuffisance de la distance entre cette dernière et l'axe de la pompe entraînerait, en effet, un 


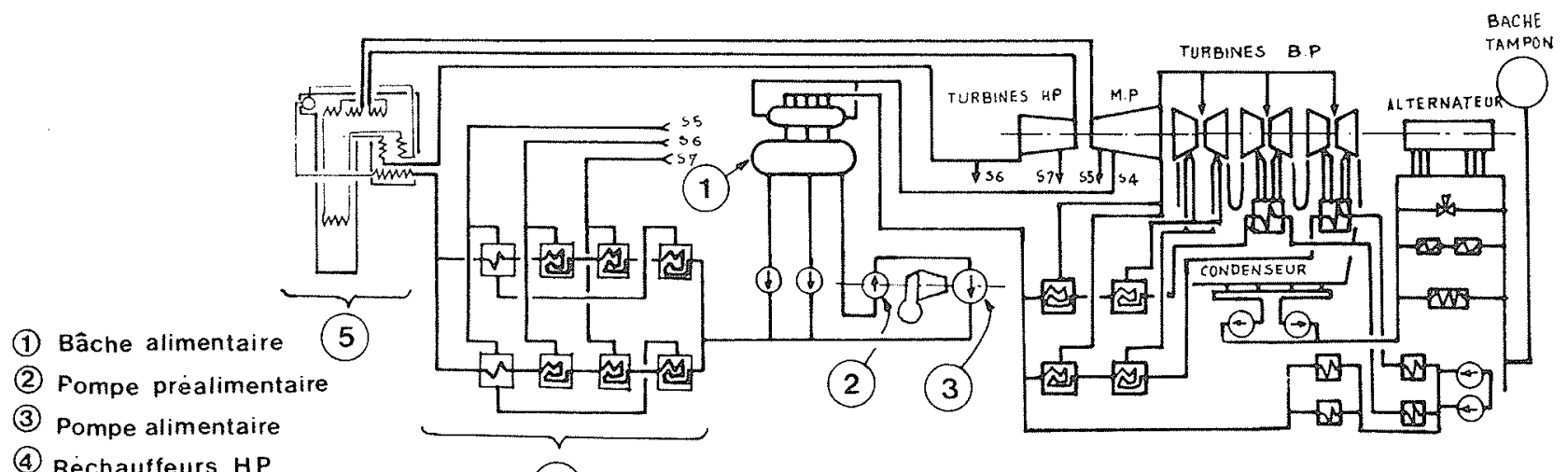

(4) Rechauffeurs HP

(5) Generateur de vapeur

(4)

Figure 1 - Schéma du poste d'eau

Lègende

Enveloppe des zones érodees

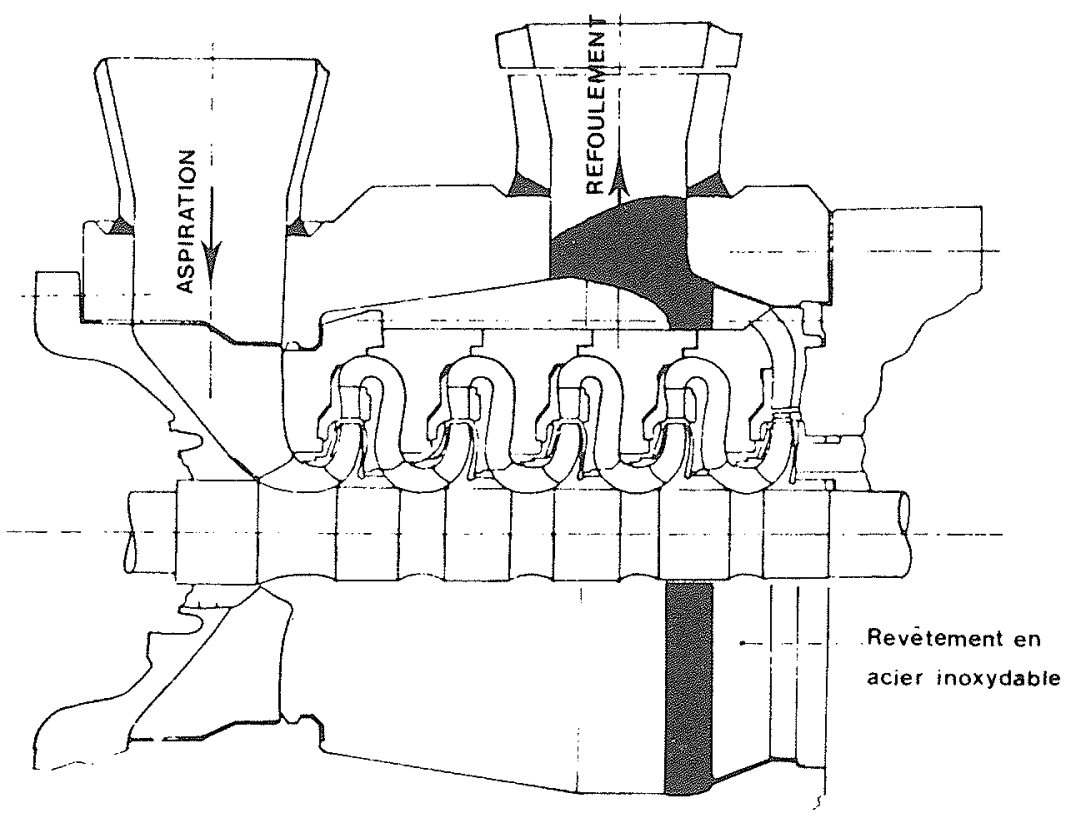

Figure 2 - Pompe alimentaire phénomène de cavitation à l'entrée dans la première roue de la pompe.

Afin d'obtenir un niveau de pression suffisant à l'entrée de la pompe, on intercale entre cette demière et la bâche alimentaire une autre pompe dite pompe préalimentaire.

Les pompes préalimentaires sont des pompes centrifuges du type à simple ou à double volute, la roue possède une ou deux ouies. Le corps est obturé à un côté par un flasque (voir fig. $3 a$ et 3b).

Aspects généraux des constatations relatives au phénomènes d'érosion-corrosion dans les pompes alimentaires et préalimentaires

Le domaine d'observation recouvre le parc des centrales thermiques classiques de $250 \mathrm{MW}$ et de $600 \mathrm{MW}$ d'Electricité de France.

L'érosion-corrosion dans les pompes alimentaires observées, se manifeste au niveau du corps de pompe, généralement conçu actuellement en acier forgé du type $\mathrm{XC} 18 \mathrm{f}$; l'enveloppe des zones fréquemment érodées inclut le voisinage du dernier diffuseur et l'entonnement de trou vers la tubulure de refoulement (voir fig. 2).

Ce phénomène apparait sous forme d'affouillements, locaux ou généralisés, pouvant atteindre $15 \mathrm{~mm}$ de profondeur à certains endroits, mais s'arrête au niveau d'un revêtement pré-existant en acier inoxydable ; il convient de noter que ce revêtement est exposé à l'écoulement dans pratiquement les mêmes conditions que l'acier forgé du corps de pompe et qu'il a toujours été découvert intact (voir fig. 2 et 4 ).

L'ordre de grandeur des vitesses d'écoulement, correspondant à la puissance nominale de la centrale, dans le voisinage des zones érodées est de $13 \mathrm{~m} / \mathrm{s}$ pour les tranches de $250 \mathrm{MW}$ et de $17 \mathrm{~m} / \mathrm{s}$ pour celles de $600 \mathrm{MW}$.

Tout comme dans les pompes alimentaires, l'érosioncorrosion dans les pompes préalimentaires se manifeste au niveau du corps de pompe, généralement conçu actuellement en acier moulé du type A $48 \mathrm{M}$, l'enve- 
a)

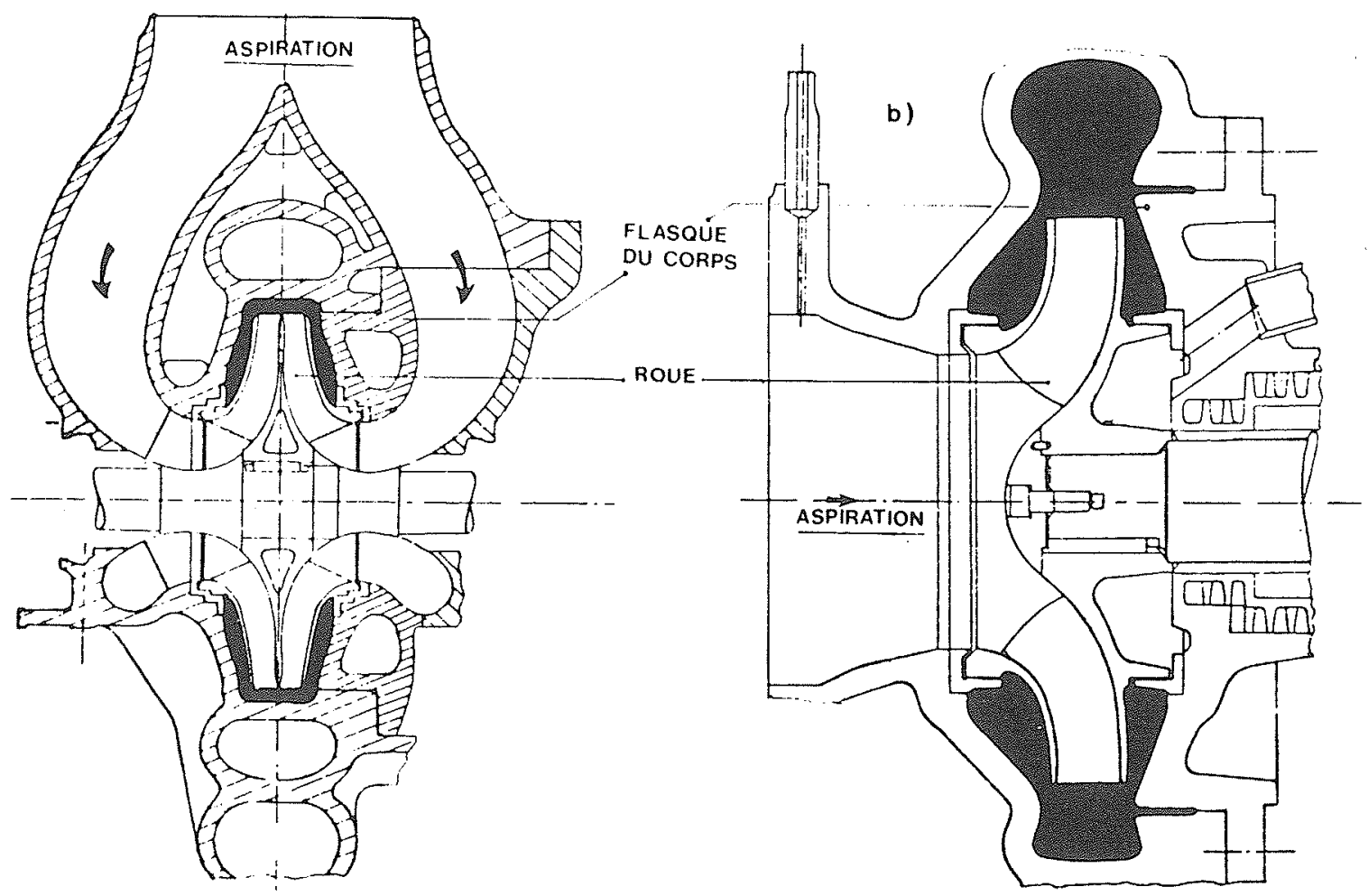

Figure 3 - Pompe préalimentaire,

$\begin{array}{ll}\text { a) A double volute, } & \text { b) A simple volute }\end{array}$

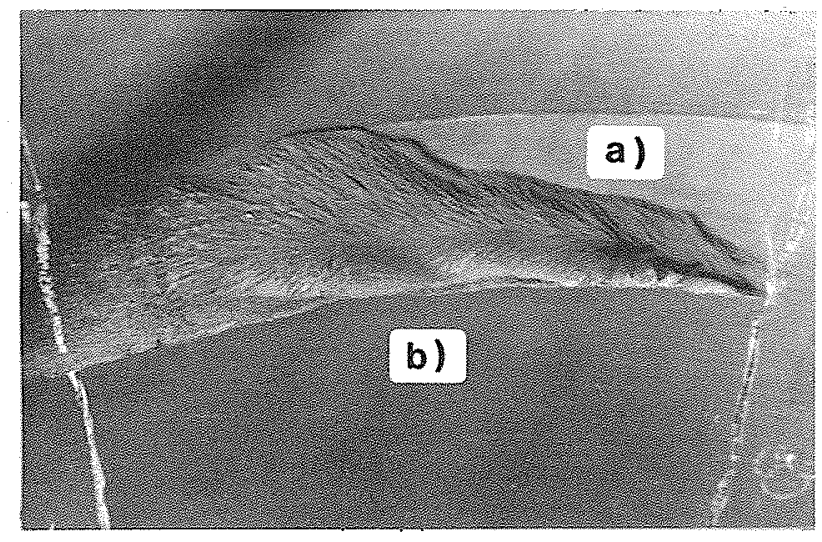

Figure 4 - Détail d'une zone érodée dans le voisinage du revêtement en acier inoxydable, a) Acier forgé, b) Revêtement en acier inoxydable

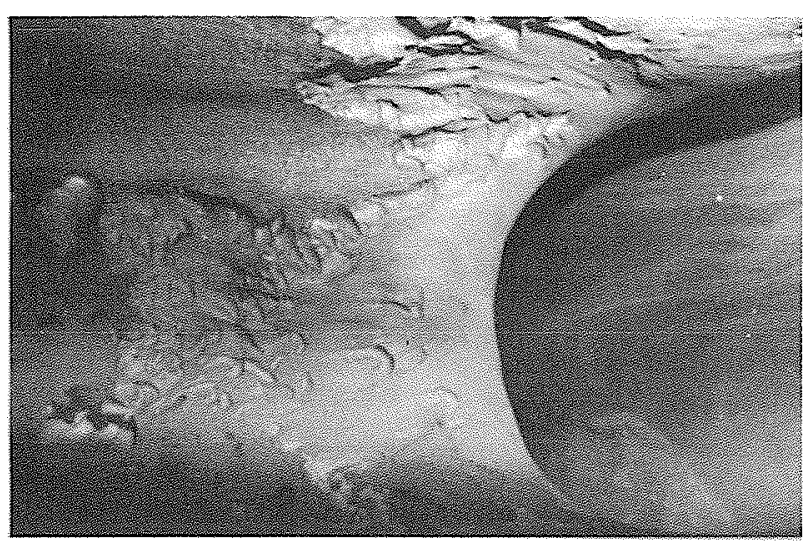

Figure 5 - Bec de volute

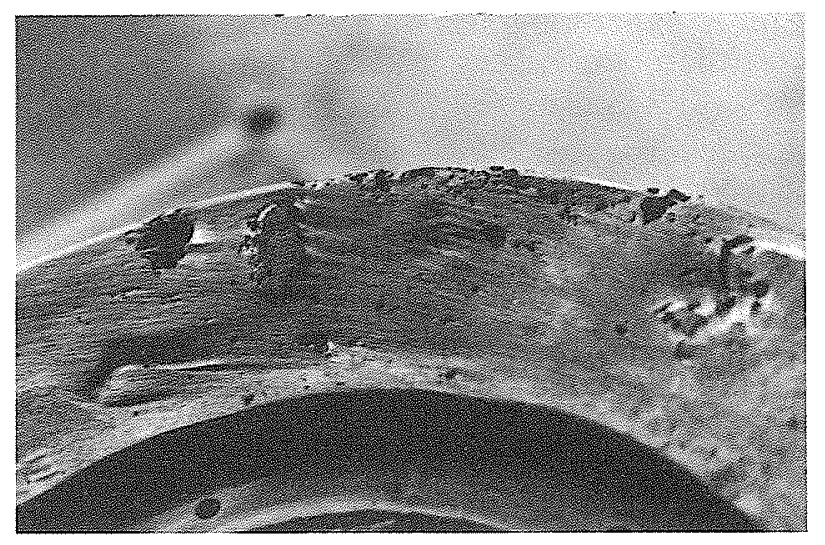

Figure 6 - Détail d'une zone érodée du flasque du corps

loppe des zones fréquemment érodées inclut la volute dans sa totalité et le flasque du corps (voir fig. 3).

L'érosion-corrosion apparaît sous forme d'usures généralisées de 4 à $5 \mathrm{~mm}$ de profondeur avec, à certains endroits, notamment dans le voisinage du bec de volute, des affouillements pouvant atteindre 10 à $15 \mathrm{~mm}$ de profondeur (voir fig. 5 et 6 ).

L'ordre de grandeur des vitesses d'écoulement est pratiquement le même pour les tranches de $250 \mathrm{MW}$ et de $600 \mathrm{MW}$; il est de $23 \mathrm{~m} / \mathrm{s}$ dans le voisinage du bec de volute, de $13 \mathrm{~m} / \mathrm{s}$ à $23 \mathrm{~m} / \mathrm{s}$ dans la volute et de 9 à $19 \mathrm{~m} / \mathrm{s}$ dans le voisinage du flasque du corps. 


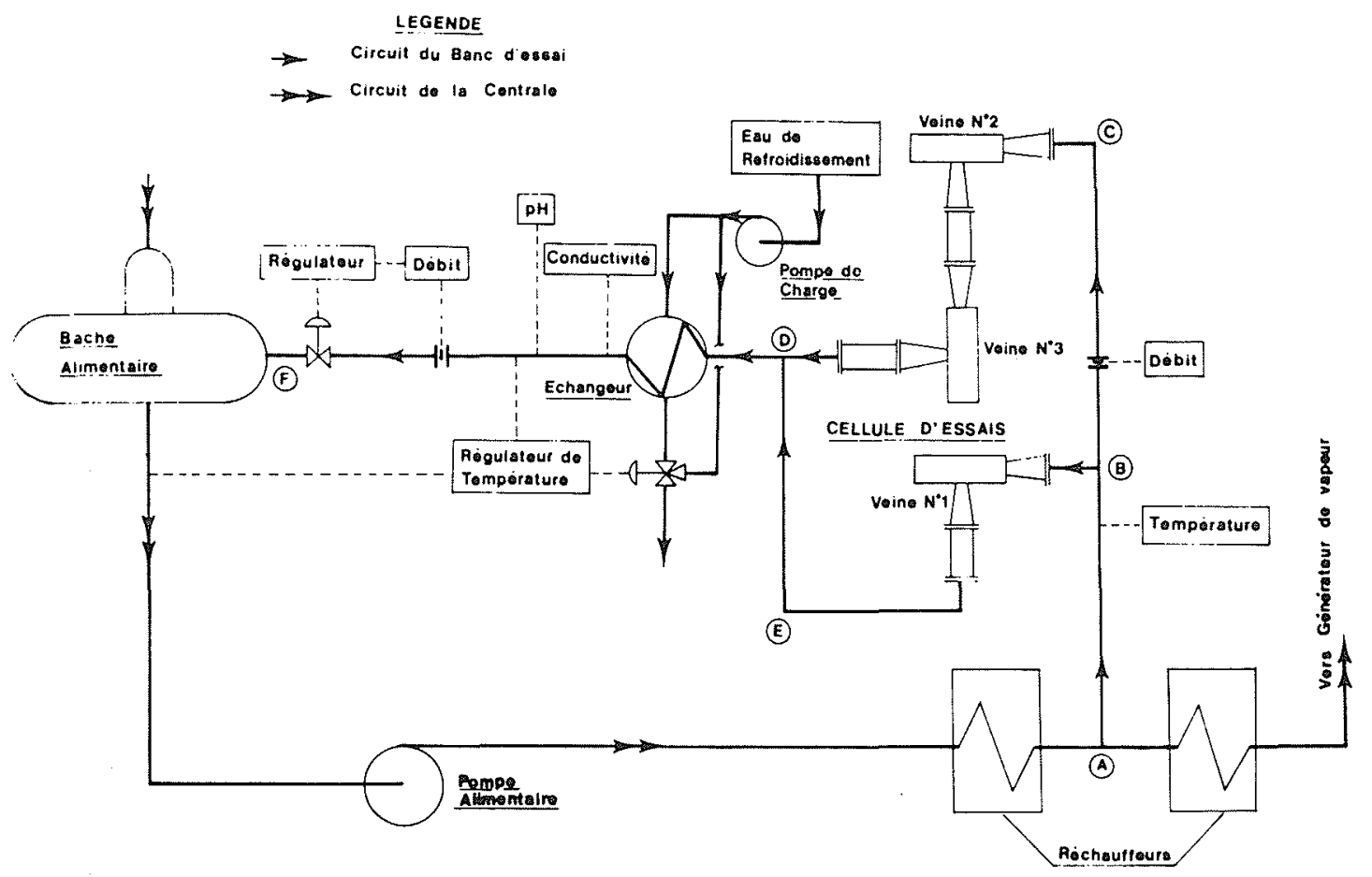

Figure 7 - Schéma de principe de la boucle d'essai d'érosion-corrosion

Etudes expérimentales du phénomène d'érosioncorrosion envisagées à Electricité de France

\section{A - Boucle d'essai d'érosion-corrosion au Département Machines et Structures (EDF-DER Chatou)}

Les études systématiques sur l'influence des grandes vitesses d'écoulement sur la vitesse de corrosion des matériaux qui sont, ou peuvent être, utilisés sur les pompes à vitesse de rotation rapide sont relativement rares à l'heure actuelle.

Aussi la Direction des Etudes et Recherches d'Electricité de France a-t-elle entrepris la construction d'une boucle d'essai permettant d'étudier le comportement de certains matériaux constitutifs des pompes alimentaires des centrales thermiques classiques et nucléaires, vis-àvis du phénomène d'érosion-corrosion dans l'eau chaude à grande vitesse d'écoulement $(80 \mathrm{~m} / \mathrm{s}$ et plus).

\section{Description sommaire de la boucle d'essai}

La boucle d'essai est montée en dérivation sur un circuit d'alimentation d'une centrale thermique classique conformément au schéma de principe de la figure 7 .

Le fluide est de l'eau d'alimentation du générateur de vapeur prélevée à la sortie du premier réchauffeur $\mathrm{HP}$ situé en aval des pompes alimentaires, à une température de $205^{\circ} \mathrm{C}$ et à une pression de $118 \mathrm{bar}$.

Cette eau, après passage dans la cellule d'essai (qui fait aussi office de déchargeur) traverse un échangeur avant sa réinjection dans la bâche alimentaire à une température de l'ordre de $150^{\circ} \mathrm{C}$ et à une pression de $5 \mathrm{bar}$.

L'organe principal de la cellule d'essai est un dispositif hydrodynamique de veine vortex (voir fig. $8 \mathrm{a}$ et $8 \mathrm{~b}$ ).

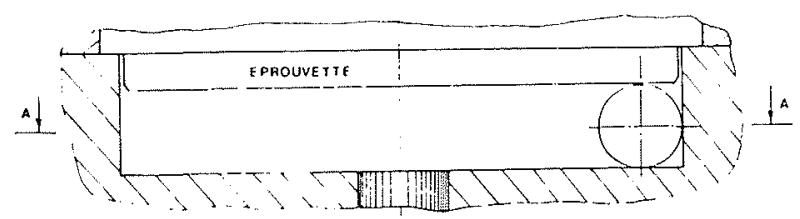

2) COUPE B.B
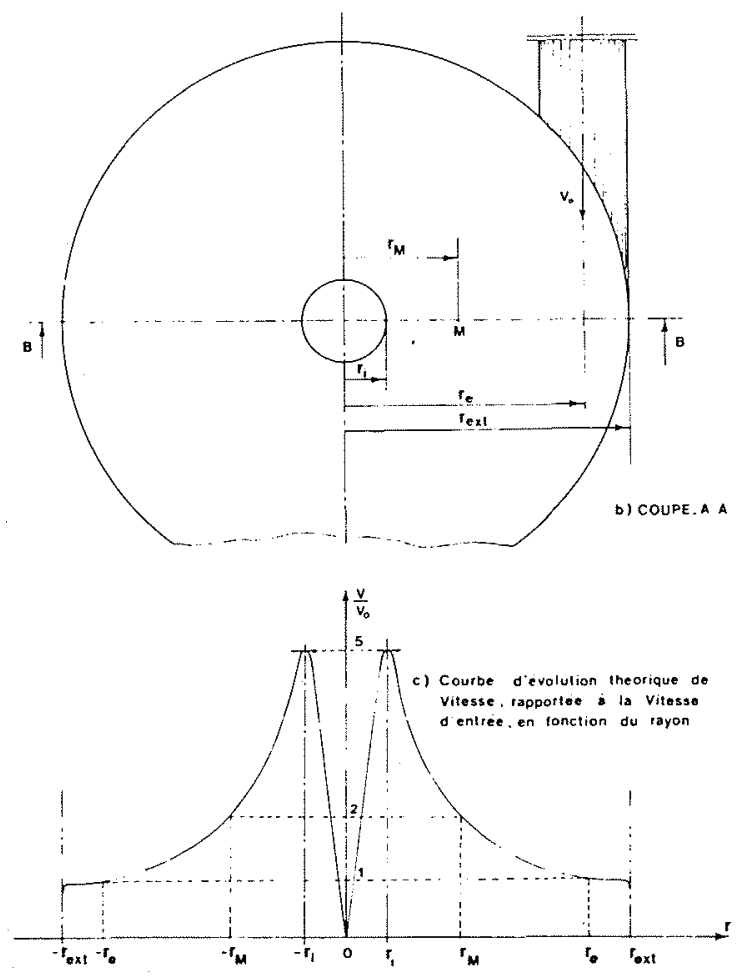

Figure 8 - Veine d'essai 
Son intérêt principal est, d'une part, de permettre la génération d'une répartition continue de vitesse très étendue au cours d'un même essai, et d'autre part, son encombrement réduit et sa simplicité.

Chaque dispositif se compose (voir figures $8 \mathrm{a}$ et $8 \mathrm{~b}$ ) d'un boîtier cylindrique avec un raccordement tangentiel, par lequel s'effectue l'arrivée du fluide, et un autre axial pour son évacuation ; le fond du boittier, exposé à l'écoulement en rotation, est amovible et constitue l'éprouvette d'essai. Une ouverture cylindrique pratiquée dans le couvercle du boîtier permet de suivre, à l'aide d'un appareil à ultrasons, l'évolution dans le temps de l'ordre de grandeur des pertes de métal.

Dans une telle configuration, la composante tangentielle de la vitesse d'écoulement crô̂t au fur et à mesure que l'on s'approche de la conduite d'évacuation du fluide, passe par un maximum (de l'ordre de $100 \mathrm{~m} / \mathrm{s}$ pour un débit de $20 \mathrm{t} / \mathrm{h}$ ) dans le voisinage de cette dernière et décroît jusqu'à une valeur nulle au centre du boîtier (voir fig. $8 \mathrm{c}$ ).

Un dispositif de ce type a fait l'objet d'une mise au point à caractère expérimental, sur modèle transparent, et à caractère théorique.

\section{$B-$ Boucle d'essai d'érosion-corrosion aux Départe- ments Etudes de matériaux et Technologie des com- posants (EDF-DER Les Renardières)}

Les essais ont pour but d'étudier l'influence des paramètres physico-chimiques de l'eau (type de conditionnement $-\mathrm{pH}-$ température) sur l'érosion-corrosion des aciers soumis à de grandes vitesses d'écoulement.

Description sommaire de la boucle d'essai (voir fig. 9)

De l'eau désaérée en provenance d'un circuit de traitement est conditionnée dans un bac pour obtenir le $\mathrm{pH}$ souhaité (à l'aide d'ammoniaque ou de morpholine) puis refoulée dans une bâche de réserve.

Deux pompes volumétriques aspirent l'eau de la bâche et refoulent un débit total de $600 \mathrm{~kg} / \mathrm{h}$ sous une pression de 200 bar.

Cette eau est chauffée dans un système de tube préchauffeur, pour obtenir la température désirée, et traverse la cellule d'essai.

L'eau traverse ensuite un échangeur où elle est refroidie jusqu'à $80^{\circ}$ avant sa réinjection dans la bâche de réserve.

La cellule d'essai est constituée de quatre dispositifs à jet disposés en série. Les éprouvettes d'essai sont montées en aval immédiat de chaque jet. La face de chaque éprouvette exposée au jet est taillée en biseau. $\mathrm{La}$ vitesse maximale pouvant être réalisée est de l'ordre de $80 \mathrm{~m} / \mathrm{s}$

\section{Conclusion}

L'exposé sommaire concernant les dégradations imputables à l'érosion-corrosion dans les pompes alimentaires et préalimentaires des centrales thermiques classiques a

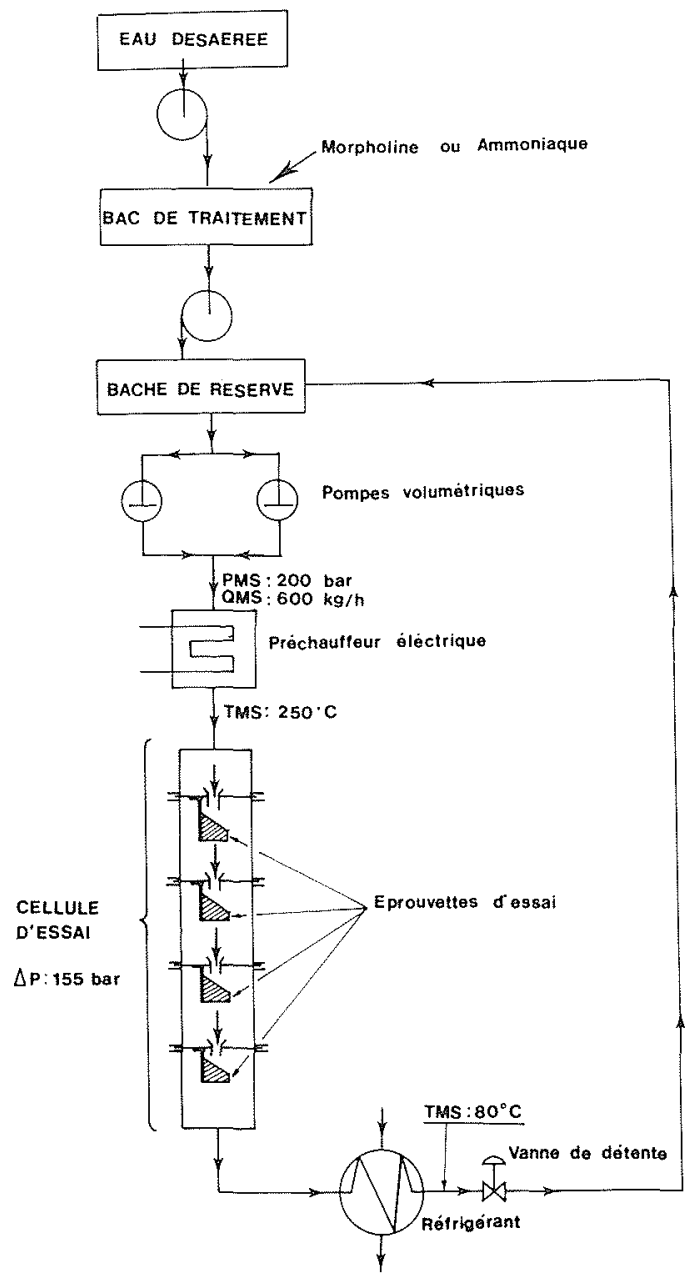

Figure 9 - Schéma de principe de la boucle d'essai d'érosion. corrosion

permis de juger de l'importance de ce phénomène dans ce type de matériel. Il est à souligner notamment les pertes d'épaisseur qui peuvent atteindre $15 \mathrm{~mm}$ à certains endroits du corps de pompe, et ceci, au bout de quelque 30000 à 40000 heures de fonctionnement et pour des vitesses d'écoulement ne dépassant pas $23 \mathrm{~m} / \mathrm{s}$. Il est intéressant de noter que la présence de chrome dans un matériau peut ralentir très nettement sa vitesse de corrosion ; c'est ainsi que dans toutes les observations que nous avons pu effectuer, les éléments de pompes en acier inoxydable, bien que soumis aux mêmes conditions chimiques, thermodynamiques et hydrauliques que ceux. en acier au carbone, se sont nettement mieux comportés que ces derniers.

Par ailleurs, les études expérimentales du phénomène d'érosion-corrosion que nous envisageons actuellement à Electricité de France, devraient permettre une meilleure connaissance du comportement des matériaux utilisés ou utilisables sur les pompes alimentaires des générateurs de vapeur et par la suite, un meilleur choix des solutions permettant de résoudre économiquement le problème d'érosion-corrosion. 


\title{
Discussion
}

\author{
Président : M. J. CHEVALIER
}

M. le Président remercie M. RANDRIANARIVO.

$\mathrm{Y}$-a-t-il des questions sur ces problèmes d'érosion-corrosion?

Je rappelle qu'on vous a parlé de vitesses élevées : $80 \mathrm{~m} / \mathrm{sec}$. mais je rappelle aussi qu'on constate également ce phénomène dans des réchauffeurs d'eau où les vitesses sont de 2 ou $3 \mathrm{~m} / \mathrm{sec}$.

M. GILMER. - On l'a vu aussi dans des tuyauteries, avec l'ordre de $7 \mathrm{~m} / \mathrm{sec}$; dans certaines centrales, à proximité notamment des vannes, mais en amont des vannes et non en aval. On pourrait croire que cela vient de l'aval, mais, en amont des vannes, on peut voir certaines corrosions de l'ordre de plusieurs centimètres, avec des vitesses de l'ordre de 6 à $7 \mathrm{~m} / \mathrm{sec}$.

M. RANDRIANARIVO. - Pour compléter la remarque de Monsieur GILMER, il convient de noter que le phénomène d'érosion-corrosion se produit dès l'instant où une vitesse relative métal-fluide existe surtout dans les conditions de température rencontrées dans les centrales.

M. le Président. - Si vous choisissez des vitesses aussi élevées que $80 \mathrm{~m} / \mathrm{sec}$., je présume que c'est pour accélérer le phénomène dans vos expérimentations?

M. RANDRIANARIVO. - Le choix des $80 \mathrm{~m} / \mathrm{sec}$. comme valeur maximale de la vitesse d'écoulement dans notre veine d'essai répond effectivement à un souci d'accélérer le phénomène d'érosion-corrosion mais aussi, et surtout, pour simuler les vitesses d'écoulement qui ne tarderont pas à apparaître dans les pompes alimentaires étant donnée l'évolution sans cesse croissante des caractéristiques de ce type de matériel.

M. le Président. - La difficulté est d'être sûr qu'il n'y aura pas apparition de phénomènes de cavitation.

M. RANDRIANARIVO. - L'apparition de phénomènes de cavitation đans la veine est théoriquement exclue étant données les conditions de pression et de température dont on dispose en amont de la veine ; cet état de fait est d'ailleurs confirmé par les résultats des essais en modèle transparent qui ont été entrepris pour définir la veine d'essai.

M. MOUGEY. - Pour répondre à la question de M. CHEVALIER, je voudrais préciser que la valeur de $80 \mathrm{~m} / \mathrm{sec}$ est la valeur maximale de la vitesse dans la cellule d'essai, qui variera de façon continue de $15 \mathrm{~m} / \mathrm{sec}$ à $80 \mathrm{~m} / \mathrm{sec}$.

Par ailleurs, il faut souligner la complémentarité des expérimentations faites sur la boucle des Renardières d'une part, et sur la boucle de Creil d'autre part. Sur la première, il est essentiellement prévu de déterminer l'influence des paramètres physicochimiques de l'eau en les faisant varier, alors que sur la seconde, c'est le paramètre vitesse d'écoulement qui est étudié, la nature de l'eau étant celle du circuit alimentaire de la centrale.

M. CANAVELIS. - Je voudrais poser deux questions : 1) $Y$-a-t-il érosion-corrosion en eau froide dans les machines hydrauliques?

2) Peut-il y avoir influence de l'abrasion par les oxydes en suspension comme semblerait l'indiquer la présence de "coups de gouge"?

M. CERDAN. - Je voudrais souligner que les "coups de gouge" ont été également constatés sur des corps de combineurs de pression immédiatement à la sortie du générateur de vapeur où la qualité est sévèrement contrôlée. On ne peut donc pas parler d'abrasion dans ces cas-là. Mais le type d'attaque avec coups de gouge est caractéristique.

M. le Président. - Je peux répondre à M. CANAVELIS en ce qui concerne sa première question : pour qu'il y ait érosioncorrosion, il faut qu'il y ait de l'eau chaude.

M. CANAVELIS. - Il y a déjà eu des corrosions hors zone de cavitation des diffuseurs de pompes en France ou à l'étranger. Peut-on considérer que c'est de l'érosion-corrosion?

M. CERDAN. - L'érosion-corrosion à laquelle on s'intéresse actuellement s'accompagne d'un passage en solution des ions ferreux dans l'eau; ce passage en solution atteint un maximum à la température de $180^{\circ}$, mais décroît très vite au-delà et endeçà, c'est-à-dire qu'aux températures voisines de la température ordinaire, les risques sont pratiquement nuls.

M. BONNAFOUX. - Pour répondre à la question posée par M. CANAVELIS je ne pense pas que dans les centrales hydrauliques d'EDF, on ait observé de phénoménes d'érosion-corrosion en eau froide. Les dégâts Ielevés ici ou là sont essentiellement des phénomènes d'abrasion par de l'eau à grande vitesse chargée de sable, par exemple dans les injecteurs de turbines

M. LACOSTE. - C'est exact. En particulier, dans les Pyrénées, on ne constate pratiquement pas d'érosion sur les turbines. L'érosion s'accompagne toujours de transports solides.

M. GUITON. - Comme facteur intervenant dans l'érosioncorrosion et indépendamment de la vitesse d'écoulement, ne peut-on pas penser qu'in tervient quelque chose que l'on pourrait appeler la densité ou l'in tensité des mèches tourbillonnaires? On peut supposer en effet que le passage de mèches tourbillonnaires au-dessus de la couche d'oxyde produit une succession de dépressions brutales sur celle-ci.

M. BERGERON. - Cela ne produirait pas des coups de gouge ; cela ferait, comme la cavitation, une sorte d'éponge. Comme l'a dit M. CANAVELIS, les coups de gouge in terviennent lorsqu'il y a des corps en suspension; tandis que la cavitation provoque des trous.

M. CERDAN. - La réciproque peut exister.

M. BERGERON, - M. CANAVELIS a dit aussi qu'il y avait parfois des oxydes de fer dans de l'eau qui avait été épurée. M. BONNIN. - Dans ce cas, il y a aussi corrosion.

M. RANDRIANARIVO. - Pour compléter les réponses à la deuxième question de M. CANAVELIS, j'ajouterai que les difféIentes théories qui ont été avancées pour expliquer le phénomène d'érusion-corrosion ont écarté l'influence des particules d'oxyde en suspension, bien qu'il ne soit pas possible a priori d'en faire abstraction; ce qui paraît cependant admis c'est que ces effets semblent être moins importants que ceux consécutifs aux autres paramètres tels que la nature du matériau, la vitesse d'écoulement, le $\mathrm{pH}$ et la température du milieu réactionnel.

En présence d'un écoulement d'eau chaude dégazée, certaines théories formulent la vitesse de corrosion des aciers sous la forme :

$$
\frac{d C}{d t}=A \cdot \sqrt{V} \cdot p\left(H_{2}\right)^{1 / 3} \cdot \sum_{b=0}^{3} k_{b}\left[H^{+}\right]^{(2-b)}
$$

dans laquelle :

A est une constante fonction du matériau

$V \quad$ la vitesse d'écoulement

$p\left(\mathrm{H}_{2}\right)$ la pression partielle en hydrogène

$k_{b}$ une constante d'équilibre du fer soluble (du type $k_{b} \quad F_{e}(O H)(2-b)$ ) avec la magnétite

$\left[H^{+}\right]$la concentration en ions $H^{+}$

Il est intéressant de noter que cette formulation traduit une loi d'oxydation du type linéaire, ce qui veut dire, en d'autres termes, que les produits de corrosion susceptibles de contribuer à la génération d'une couche d'oxyde protectrice partent in tégralement vers la solution conduisant ainsi à une dégradation très rapide du matériau (cas de l'acier noir) pouvant aller jusqu'au percement dans le cas des tubes. Dès lors, les érosions en forme de "coups de gouge", de "nids de poule", ou de "demi-œuf de pigeon" peuvent s'expliquer par l'existence d'un certain nombre de mèches tourbillonnaires engendrant des survitesses locales consécutives aux conditions génératrices de l'écoulement.

$M$. le Président. - Je remercie M. RANDRLANARIVO de ses explications. 
Vous voyez que le sujet était vaste, et j'incite ceux d'entre yous qui s'y intéressent à prendre contact avec M. CERDAN et son Groupe de travail pour essayer d'apporter un peu d'eau à ce moulin.

M. GILMER. - Je voudrais parler du phénomène électrolytique, qui n'a pas été évoqué, mais peutêtre M. CERDAN l'a-t-il fait sciemment.

Dans tous ces phénomènes d'érosion-corrosion, il y a des possibilités d'attaque électrolytique, puisque les zones d'érosioncorrosion se trouvent dans une région où il y a des rotations de mobiles de pompes qui sont en chrome en face de pièces qui sont en acier ordinaire. Dans les volutes, dans les zones qui se trouvent cachées, dans ces parties en chrome, on trouve des états de surfaces qui ne sont absolument pas attaquées.

D'autre part, on a pu voir aussi, lors des présentations de diapositives, des attaques qui se font encore plus nettement dans les zones où il y a des revêtements inoxydables. On a d'ailleurs trouvé des affouillements en-dessous de ces parties en inox. Et lorsque je parlais de tuyauteries attaquées sur plusieurs millimètres dans des zones où la vitesse d'eau avoisinait quelques mètres seulement, on voyait aussi nettement des affouillements en-dessous des parties stellitées des obturateurs de vannes ou des sièges de vannes. On voyait nettement des attaques électrolytiques et des profondeurs d'attaques dans ces zones.
M. RANDRIANARIVO. - C'est finalement une érosioncorrosion par pile.

M. GILMER' - Je me demande s'il n'y a pas aussi ces questions-là dans les pompes alimentaires.

M. CERDAN. - Vous avez tout à fait raison; dans la mesure où l'érosion-corrosion s'accompagne d'un passage en solution, il y a un phénomène électrolytique. Nous avons constaté que dans des tuyauteries où, à titre de réparation temporaire, on avait mis en place des revêtements, ceux-ci ne faisaient qu'accélérer l'érosion-corrosion sur la partie métal qui n'était pas protégée par ces revêtements. Cela ne veut pas dire qu'il soit nécessaire qu'il y ait cet effet de pile pour qu'il y ait érosion-corrosion ; on constatait l'érosion dans des zones où il y avait de l'acier noir sur une centaine de mètres.

M. CASACCI. - Quand il y a une zone protégée à côté, la corrosion ou la cavitation ou la destruction ou l'érosion va beaucoup plus rapidement.

M. le Président. - S'il n'y a plus d'autre question sur ce sujet, je remercie M. CERDAN et M. RANDRIANARIVO. Le sujet est très intéressant ; il laisse la porte ouverte à de nombreuses spéculations. 